\title{
Loss or major reduction of umami taste sensation in pinnipeds
}

\author{
Jun J. Sato • Mieczyslaw Wolsan
}

Received: 17 April 2012 / Revised: 21 June 2012 / Accepted: 23 June 2012 / Published online: 10 July 2012

(C) The Author(s) 2012. This article is published with open access at Springerlink.com

\begin{abstract}
Umami is one of basic tastes that humans and other vertebrates can perceive. This taste is elicited by Lamino acids and thus has a special role of detecting nutritious, protein-rich food. The T1R1 + T1R3 heterodimer acts as the principal umami receptor. The T1R1 protein is encoded by the Tas $1 r 1$ gene. We report multiple inactivating (pseudogenizing) mutations in exon 3 of this gene from four phocid and two otariid species (Pinnipedia). Jiang et al. (Proc Natl Acad Sci U S A 109:4956-4961, 2012) reported two inactivating mutations in exons 2 and 6 of this gene from another otariid species. These findings suggest lost or greatly reduced umami sensory capabilities in these species. The widespread occurrence of a nonfunctional Tas1r1 pseudogene in this clade of strictly carnivorous mammals is surprising. We hypothesize that factors underlying the pseudogenization of Tas $1 r 1$ in pinnipeds may be driven by the marine environment to which these carnivorans (Carnivora) have adapted and may include: the evolutionary change in diet from tetrapod prey to fish and cephalopods (because cephalopods and living fish contain little or no synergistic inosine $5^{\prime}$-monophosphate that greatly enhances umami taste), the feeding behavior of swallowing food whole without mastication (because the T1R1 + T1R3 receptor is
\end{abstract}

Communicated by: Sven Thatje

\section{J. J. Sato}

Laboratory of Animal Cell Technology, Faculty of Life Science and Technology, Fukuyama University,

Higashimura-cho, Aza, Sanzo, 985,

Fukuyama 729-0292, Japan

e-mail: jsato@bt.fubt.fukuyama-u.ac.jp

M. Wolsan $(\bowtie)$

Museum and Institute of Zoology, Polish Academy of Sciences, Wilcza 64,

00-679 Warszawa, Poland

e-mail:wolsan@miiz.waw.pl distributed on the tongue and palate), and the saltiness of sea water (because a high concentration of sodium chloride masks umami taste).

Keywords Diet $\cdot$ Feeding behavior $\cdot$ Feeding ecology . Marine adaptation $\cdot$ Marine environment

\section{Introduction}

The sense of taste is a chemosensory system that identifies nutritionally relevant and harmful substances in food and thus guides organisms to consume or avoid potential food sources. Humans and other vertebrates can detect several taste qualities, including bitter, salty, sour, sweet, and umami. The taste of umami (which means "delicious flavor") allows the recognition of L-amino acids, the building blocks of proteins, and therefore plays a special role in identifying nutritious food (Chandrashekar et al. 2006; Bachmanov and Beauchamp 2007; Temussi 2009; Yarmolinsky et al. 2009). A conspicuous feature of umami taste is its impressive potentiation by purine nucleotides such as inosine 5'-monophosphate (IMP) and guanosine 5'-monophosphate (GMP; Kuninaka 1960). Several candidate umami receptors have been proposed, including mGluR4 (Chaudhari et al. 2000), T1R1 + T1R3 (Li et al. 2002; Nelson et al. 2002), and mGluR1 (San Gabriel et al. 2005). The T1R1 + T1R3 heterodimer responds to a broad spectrum of L-amino acids (Nelson et al. 2002), shows a strongly potentiated response in the presence of IMP or GMP (Li et al. 2002; Nelson et al. 2002), and is widely regarded as the prototypic umami receptor (Temussi 2009). This receptor is embedded in the membrane of taste receptor cells, which are assembled into taste buds distributed in papillae of the tongue and palate epithelium (Chandrashekar et al. 2006; Yarmolinsky et al. 2009). The T1R1 and T1R3 proteins are encoded by the Taslr1 (TASIRl) and Tas $1 r 3$ 
(TAS1R3) genes, respectively (Bachmanov and Beauchamp 2007), the latter gene being also expressed in a T1R2 + T1R3 heterodimer, which functions as a sweet receptor (Nelson et al. 2001; Li et al. 2002).

Pseudogenization is an evolutionary phenomenon in which a gene loses its function due to a disruption in its coding or regulatory sequence (Grus and Zhang 2008). Frameshift insertions, frameshift deletions, and nonsense substitutions introduce stop codons that disrupt the open reading frame of a gene and thereby cause a premature termination of translation of nucleic acids into protein. If such a mutation occurs in Tas1r1, this gene will become a pseudogene and will no longer be able to produce a complete functional T1R1 protein. This will result in a dysfunctional T1R1 + T1R3 receptor and can eventually lead to the loss of umami perception. Most vertebrates appear to have a functional (intact) Tas1r1 (Shi and Zhang 2006; Nei et al. 2008). This gene is known to be inactivated by pseudogenization or absent in the tongueless western clawed frog (Shi and Zhang 2006); some fructivorous, insectivorous, and vampiric bats (Zhao et al. 2012); folivorous giant panda (Li et al. 2010; Zhao et al. 2010); and two marine carnivorous mammals (bottlenose dolphin and California sea lion; Jiang et al. 2012).

Here, we report multiple pseudogenizing mutations in exon 3 of Tas $1 r 1$ from six species of Pinnipedia. Jiang et al. (2012) reported two other pseudogenizing mutations in exons 2 and 6 of this gene from another pinniped species (California sea lion). These findings suggest loss or major reduction of umami sensation in these species. As pinnipeds are strictly carnivorous and entirely depend on a diet rich in proteins, the widespread occurrence of a Tas1r1 pseudogene in this clade is not expected. We hypothesize that factors underlying the pseudogenization of Taslr1 in these secondarily adapted marine carnivorans (Carnivora) may be driven by the marine environment.

\section{Materials and methods}

Partial DNA sequences of Tas $1 r 1$ exon 3 were newly determined for six pinnipeds and six other carnivorans. These sequences have been deposited in the DDBJ/EMBL/GenBank databases with accession numbers AB697513AB697524. Homologous sequences for two other carnivorans, a polar bear (HM468451) and a domestic dog (HM468447), were obtained from the DDBJ/EMBL/GenBank databases (Fig. 1).

Total genomic DNA was extracted from either tissue or blood using a standard phenol-chloroform method or a DNeasy Blood and Tissue Kit (Qiagen). Amplification was performed through PCR reactions with a KOD-plus-Neo
DNA polymerase kit (Toyobo) in an automated thermal cycler (model PC 302, Astec). Each PCR mix contained

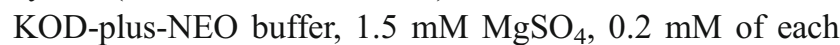
dNTP, $0.3 \mu \mathrm{M}$ of each of Tas1r1 ex3 Fw1 (5'-GGAGT GAAGCGGTATTACCC-3') and Tas1r1_ex3_Rv1 (5'GGCCTCTTCAAACTCCTTCAGGC-3') primers (both newly designed), 1.0 U of KOD-Plus-Neo DNA polymerase, and $0.1-0.2 \mu \mathrm{g}$ of template total genomic DNA in a total volume of $50 \mu \mathrm{l}$. The PCR thermal cycling parameters included a 2-min denaturation period at $94{ }^{\circ} \mathrm{C}$ followed by 35 cycles of denaturation at $98{ }^{\circ} \mathrm{C}$ for $10 \mathrm{~s}$, annealing at $50{ }^{\circ} \mathrm{C}$ for $30 \mathrm{~s}$, and extension at $68{ }^{\circ} \mathrm{C}$ for $30 \mathrm{~s}$; this was followed by a 10 -min extension period at $68{ }^{\circ} \mathrm{C}$. Sequencing reaction was carried out with a Big Dye Terminator (v. 3.1) Cycle Sequencing Kit (Applied Biosystems). Sequences were resolved using an ABI3130 automated sequencer (Applied Biosystems).

Multiple sequence alignment was accomplished in MEGA 5 (Tamura et al. 2011). Cladistic analysis was conducted in GARLI 2.0 (Zwickl 2006) using maximum likelihood with the HKY $+\Gamma$ model of DNA substitution. This optimal model was identified with the Akaike information criterion implemented in Modeltest 3.7 (Posada and Crandall 1998). Insertions were not considered. Deletions and nonsense substitutions were coded as missing data. Both canids (domestic dog and red fox) were used as an outgroup. Tree searching was heuristic. Five runs of the genetic algorithm were performed, each with 50,000 generations of a mutationselection-reproduction cycle. Starting trees were generated through stepwise addition. Bootstrap percentages were computed from 1,000 pseudoreplicates.

\section{Results}

Examination of a 453-bp aligned segment of Tas $1 r 1$ exon 3 revealed a disrupted open reading frame in all six pinnipeds and the giant panda, and an intact open reading frame in seven other carnivorans. The pseudogenizing mutations observed in the pinnipeds included a 4-bp frameshift insertion (positions 188-191; Fig. 1) in four phocids (spotted seal, harbor seal, Caspian seal, and northern elephant seal), a 1-bp frameshift deletion (position 61) and a nonsense substitution (position 248) in one phocid (northern elephant seal), and a nonsense substitution (position 61) in two otariids (Australian sea lion and South American sea lion). A 2-bp frameshift insertion previously reported from the giant panda ( $\mathrm{Li}$ et al. 2010; Zhao et al. 2010) was confirmed (positions 297298). Each of these mutations introduced a premature stop codon (Fig. 1). Phylogenetic analysis demonstrated that the 4-bp insertion first occurred in a common ancestor of the four phocids and was followed by the 1-bp deletion and nonsense substitution in the northern elephant seal lineage, 
Spotted seal Phoca largha Harbor seal Phoca vitulina Caspian seal Pusa caspica Northern elephant seal Mirounga angustirostris Australian sea lion Neophoca cinerea South American sea lion Otaria byronia Giant panda Ailuropoda melanoleuca Polar bear Ursus maritimus Red panda Ailurus fulgens Raccoon Procyon lotor Ermine Mustela erminea Striped skunk Mephitis mephitis Domestic dog Canis familiaris Red fox Vulpes vulpes

\section{1}

$$
21
$$

31

51

61

71

81 TCTTTTCTGCTCACCATCCCCAGTGATAAGCACCAGGTGGAGATCACAGTGCTGCTGCTGCAGAGGTTTGGGTGGGTCTGG TCTTTTCTGCTCACCATCCCCAGYGATAAGCACCAGGTGGAGATCACAGTGCTGCTGCTGCAGAGGTTTGGGTGGGTCTGG TCTTTTCTGCTCACCATCCCCAGCAATAAGCACCAGGTGGAGATCACAGTGCTGCTGCTGCAGAGGTTTGGGTGGGTCTGG TCTTTTCTGTGCACCATCCCCAGCGATAAGCACCAGGTGGAGATCACAGTGCTGCTGCTG-AGAGGTTTGGGTGGGTCTGG TCCTTTCTGCGCACCATCCCYGGCGATAAGGACCAGGTGGAGATCATAGTGCTGCTGCTGIAGAGGTTTGGGTGGGTCTGG TCCTTTCTGCGCACCATCCCCGGCGATAAGCACCAGGKGGAGATCATAGTGCTGCTGCTGIAGAGGTTTGGGTGGGTCTGG TCATTTCTGCGCACCATCCCCAGCGATGAGCACCAGGTTGAGCTCATGGTGCTGCTGCTGCAGAGCTTTGGGTGGGTCTGG TCATTTCTGCGCACCATCCCCAGCGATGAGCACCAGGTTGAGCTCATGGTGCTGCTGCTGCAGAGCTTTGGGTGGGTCTGG TCGTTTCTGCGCACCATCCCCAGCGATGAGCACCAGGTGGAGGTCCTAGTGCTGCTGCTGCAGAGGTTTGGGTGGGTCTGG TCGTTTCTGCGCACCATCCCCAGCGATGAGCACCAGGTGAAGGTCCTGGTGCTGCTGCTGCAGAAGTTTGGGTGGGTCTGG TCGTTTCTGCGCACCATCCCCAGTGATGAGCACCAGGTGGAGGTCCTGGTGCTGCTGCTKCAGAGGTTTGGGTGGGTCTGG TCGTTTCTGCGCACCATCTCCAGCGATGAGCACCAGGTGGAAGTCCTAGTGCTGCTGCTGCAGAGRTTTGGGTGGGTTTGG TCGTTTCTGCGCACTATCCCCAGCGATAAGTACCAGGTGGAGATCATGGTGCTACTGCTGCAGAGGTTTGGGTGGGTCTGG TCGTTTCTGCGCACTATCCCCAGCGATAAGTACCAGGTGGAGATCATGGTGCTACTGCTGCAGAGGTTTGGGTGGGTCTGG 91 111 121 131 141 151 161 171

Spotted seal ATCTCACTGGTGGGCAGCGACGGTGACTACGGGCAGCTGGGGGTGCAGGCACTGGAGGAGCAGGCCACCCAGCAGGGCATCTGCATTGCCTTC Harbor seal ATCTCACTGGTGGGCAGCGACGGTGACTACGGGCAGCTGGGGGTGCAGGCACTGGAGGAGCAGGCCACCCAGCAGGGCATCTGCATTGCCTTC Caspian seal ATCTCACCGGTGGGCAGCGACGGTGACTACAGGCAGCTGGGGGTACAGGCACTGGAGGAGCAGGCCACCCAGCAGGGCATCTGCATTGCCTTC Northern elephant seal ATCTCACTGGTGGGCAGCAACGATGACTATGGGCAGTTGGGGGTGCAGGCACTGGAGGAGCAGGCCACCCAGCAGGGCATCTGCATTGCCTTC Australian sea lion ATCTCACTGATGGGCAGTGACGGTGACTACGGGCAGCTGGGGGTGCAGGCACTGGAGGAGCAGGCCACCCAGCAGGGCATCTGCATTGCCTTC South American sea lion ATCTCACTGATGGGCAGTGACGGTGACTATGGGCAGCTGGGGGTGCAGGCACTGGAGGAGCAGGCCACCCAGCAGGGCATCTGCATTGCCTTC Giant panda ATCTCGCTGGTGGGCAGTGACGGTGACTACGGGCAGCTGGGGGTGCAGGCGCTGGAGGAGCAGGCCACCCAGCAGGGCATCTGCATTGCCTTC Polar bear ATCTCGCTGGTGGGCAGTGACGGTGACTACGGGCAGCTGGGGGTGCAGGCGCTGGAGGAGCAGGCCACCCAGCAGGGCATCTGCATTGCCTTC Red panda ATCTCGTTGGTGGGCAGCGACGGCGACTACGGGCAGCTGGGGGTGCAGGCATTGGAGGACCTGGCCCCCCAGCAGGGCATCTGCATTGCCTTC Raccoon Striped skunk Domestic dog Red fox AT ATCTCGCTGGTGGGCAGTGAYGGCGACTACGGGCAGCTAGGGGTGCAGGCGCTGGAGGAGCTGGCCACCCAGCAGGGCATCTGCATTGCCTTC ATCTCATTGGTGGGCAGCGACGGCGACTATGGGCAGCTGGGGGTGCAGGCACTGGAGGAGCAGGCCACCCAGCAGGGCATCTGCATTGCCTTC ATCTCATTGGTGGGCAGCGACGGCGACTATGGGCAGCTGGGGGTGCAGGCACTGGAGGAGCAGGCCACCCAGCAGGGCATCTGCATTGCCTTC 181 191 201 221 231 241 251 261

Spotted seal Harbor seal Caspian seal Northern elephant seal Australian sea lion South American sea lion Giant panda Polar bear Red panda Red panda Raccoon

Ermine Striped skunk Striped skunk Red fox

AAGGACATCATACCCTTCCTTCTCTGCCCGCCCGGGGAATGAGAGGATGCAGAGCATGATGCACCACCTGGCCCGAGCGAGGACCACCGTGGTG AAGGACATCATACCCTTCCTTCTCTGCCCGCCCGGGGAATGAGAGGATGCAGAGCATGATGCACCACCTGGCCCGAGCGAGGACCACCGTGGTG AAGGACATCATACCCTTCCTTCTCTGCCCGCCCTGGGAATGAGAGGATGCAGAGCATGATGCACCACCTGGCCCGAGCGAGGACCACCGTGGTG AAGGACATCATACCCTTCCTTCTCTGCCCGCCCGGGGAATAAGAGGATGCAGAGCATGATGCACCACCTGGCCTGAGCTAGGACCACCGTGGTG AAGGACATCATAC--- CCTTCTCTGCCCGCCCGAGGAATGAGAGGATGCAGAGCATSATGCACCACCTGGCCCGAGCGAGGACCACCGTGGTG AAGGACATCATAC----CCTTCTCTGCCCGCCCGAGGAATGAGAGGATGCAGAGCATGATGCACCACCTGGCCCGAGCGAGGACCACCATGGTG AAGGACATCATAC----CCTTGTCTGCCCGCCCGGGGAGCGAGAGGATGCAGAGCATGATGCACCACCTGGCCCGAGCGAGGACCACCGTGGTG AAGGACATCATAC-- - -CCTTGTCTGCCCGCCCGGGGAGCGAGAGGATGTGGAGCATGATGCACCACCTGGCCCGAGCGAGGACCACCGTGGTG AAGGACATCATAC----CCTTCTCTGCCCACCCGGGGGATGAGAGGATGCAGGGCATGATGCTCCACCTGGCCCAAGCGAGGACCACCGTGGTG AAGGACATCATAC----CCTTCTCTGCCCACCCGGGGAATGAGAGGATGCAGGCCATGATGCTCCACCTGGCCCAAGCAAGGACCACCGTGGTG AAGGATATCATAC----CCTTCTCTGCCTACCCGGGGAGTGAGAGGATGCAGGCCATGATGCTTCACCTGGCCCGAGCGAGGACCACCGTGGTG AAGGACATCATAC----CCTTCTCTGCCCACCCGGGGAATGAGAGGATGCGGGCTATGATGCTCCACCTGGCCCGAGCGAGGACCACCGTGGTG AAGGACATCATAC----CCTTCTCTGCCCAGCCGGGTAATGAGAGGATGCAGAGCATGATGTACCACCTGGACCGAGCAAGGACCACTGTTGTG AAGGACATCATAC----CCTTCTCTGCCCAGCCGGGTAATGAAAGGATGCAGAGCATGATGTACCACCTGGACCGAGCGAGGACCACTGTTGTG 271

$$
281
$$

301

311

331

341 351

Spotted seal GTCATTTTCTCCAGCAGGCAGCTGGCCA--GGGTGTTCTTCGAGTCCGTGGTGCTGGCCAACCTGGCTGGCAAGGTGTGGATCACCTCAGAG Harbor seal Caspian seal Northern elephant seal Australian sea lion GTCATTTTCTCCAGCAGGCAGCTGGCCA--GGGTGTTCTTCGAGTCCGTGGTGCTGGCCAACCTGGCTGGCAAGGTGTGGATCACCTCAGAG GTCATTTTCTCCAGCAGGCAGCTGGCCA--GGGTGTTCTTCGAGTCCGTGGTGCTGGCCAACCTGACTGGCAAGGTGTGGATCACCTCAGAG GTCATTTTCTCCAGCAGGCAGCTGGCCA--GGGTGTTCTTCGAGTCCGTGGTGCTGGCCAACCTGACTGGCAAGATGTGGATCACCTCAGAG GTCATTTTCTCCAGCAGGCAGCTGGCCA--GGGTGTTCTTCGAGTCCGTGGTGCTGGCCAACCTGACTGCCAAGGTGTGGATCGCCTCAGAG South American sea lion GTCATTTTCTCCAGCAGGCAGCTGGCCA--GGGTGTTCTTCGAGCCCGTGGTGCTGGCCAACCTGACTGCCAAGGTGTGGATCGCCTCAGAG Giant panda GTCGTCTTCTCCAACAGGCAGCTGGCCCGGGGGGTTCTTCAAGTCCATGGTGCTGGCCAACCTGACGGCCAAGGTGTGGATCGCCTCAGAG Polar bear GTCGTCTTCTCCAACAGGCAGCTGGCCC--GGGTGTTCTTCGAGTCCGTGGTGCTGGCCAACCTGACGGCCAAGGTGTGGATCGCCTCAGAG Red panda GTYGTTTTCTCTAGCAGGCAGCTGGCCA--GGGTGTTCTTTGAGTCCGTGGTGCTGGCCAACCTCACTGCCAAGGTGTGGATTGCTTCAGAG Ermine Striped skunk Domestic dog Red fox GTCGTTTTCTCCAGCAGGCAGCTGGCCA--GGGTGTTCTTTGAGTCCGTGGTGCTGGCCAACCTCACCTCCAAGGTGTGGATCGCCTCAGAG GTCGTCTTCTCCAGCAGGCAGCTGGCCA--GGGTGTTCTTCGAGTCCGTGGTGCTGACCAACCTCACYGCCAAGGTGTGGATCGCCTCCGAG GTCGTTTTCTCCAGCAGGCAGCTGGCCA--GGGTATTCTTCGAGTCCGTGGTGTTGGCCAACCTCACTGCCAAGGTGTGGATAGCCTCAGAG GTCGTTTTCTCCAGCAGGCAGCTGGCCA--GGGTGTTCTTCGAGTCCGTGGTCCTGGCCAAGCTGACTGCCAAGGTGTGGATCGCTTCAGAA GTCGTTTTCTCCAGCAGGCAGCTGGCCA--GGGTGTTCTTCGAGTCTGTGGTCCTGGCCAAGCTGACTGCCAAGGTGTGGATCGCTTCAGAA 361
371
381
391
401
411
421
431
441
451

Spotted seal GACTGGGCCATCTCCAGACACATCAGCAGCTTGCCCGGGATCTGGGGCATTGGCACAGTGCTGGGCGTGGCCATCCAGCAGCGGCTTGTCCCT Harbor seal

Caspian seal

Northern elephant seal Australian sea lion South American sea lion Giant panda Polar bear Red panda Raccoon

Ermine

Striped skunk Domestic dog Red fox GACTGGGCCATCTCCAGACACATCAGCAGCTTGCCCGGGATCTGGGGCATTGGCACAGTGCTGGGCGTGGCCATCCAGCAGCGGCTTGTCCCT GACTGGGCCATCTCCAGACACATCAGCAGCTTGCCCGGGATCTGGGGCATTGGCACAGTGCTGGGCGTGGCCATCCAGCAGCGGCTTGTCCCT GACTGGGCCATCTCCAGACACATCAGCAGCTTGCCCGGGATCTGGGGCATTGGCACAGTGCTGGGCGTGGCCATCCAGCAGCGGCTTGTCCCT GACTGGGCCATCTCCAGACACATCAGCAGCGTGCCCGGG------ATTGGCACAGTGCTGGGTGTGGCCATCCAGCAGCGGCTTGTTCCT GACTGGGCCATCTCCAGACACATCAGCAGCGTGCCCGGG--n GACTGGGCCATCTCCAGACACATCAGCAGCGTGCCCGGGGTCTGGGGCATTGGCATGGTGCTGGGCGTGGTGATTCAGCAGAGGCTTGTCCCC GACTGGGCCATCTCCAGACACATCAGCAGCGTGCCCGGGGTCTGGGGCATTGGCATGGTGCTGGGCGTGGCCATTCAGCAGAGGCTTGTCCCT GACTGGGCCATCTCCAGACACATCAGCAGCGTGCCTGGCATCTGGGGCATTGGCACGGTGCTGGGTGTGGCCATCCAGCAGAGGCTTGTCCCT GACTGGGCCATCTCCAGACACATCAGCAACGTGCCCGGGATCTGGGGCATTGGCACGGTGCTGGGTGTGGCCATCCACCAGAGGCTTGTTCCT GACTGGGCCATCTCCAGACACATCAGCAGCGTGCCCGGGATCTGGGGCATTGGCACGGTACTGGGTGTGGCCATCCAGCAGAGGCTTGTCCCT GACTGGGGCATCTCCAGACATATCAGCAGTGTGCCCGGGATCTGGGGCATTGGCACGGTGCTGGGTGTGGCCATCCAGCAGAGGCTTGTCCCT GACTGGGCCATCTCCAGACATATTAGCAGCCTGCCCAGGATCTGGGGCATTGGCACAGTGTTGGGCGTGGCCATCCAGCAGAAGCTTGTCCCT GACTGGGCCATCTCCAGACATATTAGCAGCCTGCCCAGGATCTGGGGCATTGGCACAGTGTTGGGCGTGGCCATCCAGCAGAAGCTTGTCCCT

Fig. 1 Alignment of a DNA sequence segment from Tas $1 r 1$ exon 3 in 14 carnivorans. Inactivating (pseudogenizing) mutations are underlined. Premature stop codons introduced by these mutations are boxed.
Gray shading shows the correct open reading frame. Dashes point to alignment gaps. Numbers indicate nucleotide positions in this alignment 


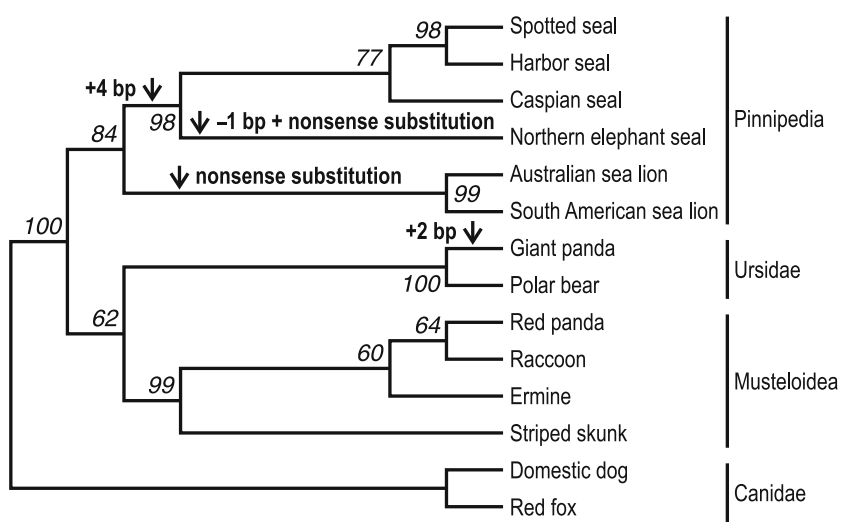

Fig. 2 Maximum likelihood phylogeny of 14 carnivorans inferred from sequences tabulated in Fig. 1. Arrows indicate branches where pseudogenizing frameshift insertions $(+4 \mathrm{bp}$ and $+2 \mathrm{bp})$, frameshift deletion $(-1 \mathrm{bp})$, and nonsense substitutions are hypothesized to have evolved. Numbers in italics represent bootstrap percentages for the respective nodes

and that the nonsense substitution found in the two otariids arose in their common ancestor (Fig. 2).

\section{Discussion}

The efficient gain of relevant nutrients and avoidance of harmful compounds are essential for the survival and propagation of organisms, and therefore, dietary, feeding behavior, and taste perception changes in organismal evolution are likely to be responded to and reflected in the evolution of genes encoding taste receptor proteins. Pinnipeds mostly feed on fish and cephalopods, and typically consume prey that is small enough to be swallowed whole (Berta et al. 2006). IMP (which greatly enhances the taste of umami) is abundant in muscles and other tissues of tetrapods, but is scarce or absent in cephalopods and living fish (Arai and Saito 1961; Arai 1966; Yamaguchi and Ninomiya 2000; Kurihara 2009). This synergistic compound gradually accumulates postmortem in fish tissues as a result of degradation of adenosine 5'-triphosphate, so that umami taste can eventually be perceived some time after the fish's death (Arai and Saito 1961; Arai 1966; Kurihara 2009). Furthermore, a high concentration of sodium chloride masks umami taste (Ikeda 1909; Komata 1990). We therefore hypothesize that factors underlying the inactivation of Tas $1 r 1$ in pinnipeds may be driven by the marine environment to which these carnivorans have adapted and may include: the evolutionary change in diet from tetrapod prey to fish and cephalopods, the feeding behavior of swallowing food whole without mastication (also suggested by Jiang et al. 2012), and the saltiness of sea water.

The ability of pinnipeds to sense umami has not been examined. Anatomical studies, however, have shown that gustatory papillae on the tongue of pinnipeds are reduced in number and simplified as compared with other carnivorans (Sonntag 1923; Kubota 1968; Yoshimura et al. 2002). These observations concur with our finding of the widespread pseudogenization of Tas $1 r 1$ in pinnipeds, which suggests lost or at least greatly reduced umami sensory capabilities.

Acknowledgments We thank Hideki Endo, Jacek Goszczyński, Tetsuji Hosoda, Masatoshi Kadokawa, Mari Kobayashi, Shinji Minami, Małgorzata Pilot, Hitoshi Suzuki, Kimiyuki Tsuchiya, Takahiro Yonezawa, and staffs in the Kamogawa Sea World for providing tissue samples. We also thank three anonymous reviewers for their comments.

Open Access This article is distributed under the terms of the Creative Commons Attribution License which permits any use, distribution, and reproduction in any medium, provided the original author(s) and the source are credited.

\section{References}

Arai K (1966) Nucleotides in the muscles of marine invertebrate animals. Bull Jpn Soc Sci Fish 32:174-180 (in Japanese)

Arai K, Saito T (1961) Changes in adenine nucleotides in the muscles of some marine invertebrates. Nature 192:451-452

Bachmanov AA, Beauchamp GK (2007) Taste receptor genes. Annu Rev Nutr 27:389-414

Berta A, Sumich JL, Kovacs KM (2006) Marine mammals: evolutionary biology, 2nd edn. Elsevier, Amsterdam

Chandrashekar J, Hoon MA, Ryba NJP, Zuker CS (2006) The receptors and cells for mammalian taste. Nature 444:288-294

Chaudhari N, Landin AM, Roper SD (2000) A metabotropic glutamate receptor variant functions as a taste receptor. Nat Neurosci 3:113-119

Grus WE, Zhang J (2008) Human lineage-specific gene inactivation. In: Encyclopedia of life sciences. Wiley, Chichester. doi:10.1002/ 9780470015902.a9780470020835

Ikeda T (1909) New seasonings. J Chem Soc Tokyo 30:820-836 (in Japanese)

Jiang P, Josue J, Li X, Glaser D, Li W, Brand JG, Margolskee RF, Reed DR, Beauchamp GK (2012) Major taste loss in carnivorous mammals. Proc Natl Acad Sci U S A 109:4956-4961

Komata Y (1990) Umami taste of seafoods. Food Rev Int 6:457-487

Kubota K (1968) Comparative anatomical and neurohistological observations on the tongue of the northern fur seal (Callorhinus ursinus). Anat Rec 161:257-265

Kuninaka A (1960) Studies on taste of ribonucleic acid derivatives. J Agric Chem Soc Jpn 34:489-492 (in Japanese)

Kurihara K (2009) Glutamate: from discovery as a food flavor to role as a basic taste (umami). Am J Clin Nutr 90:719S-722S

Li X, Staszewski L, Xu H, Durick K, Zoller M, Adler E (2002) Human receptors for sweet and umami taste. Proc Natl Acad Sci U S A 99:4692-4696

Li R, Fan W, Tian G et al (2010) The sequence and de novo assembly of the giant panda genome. Nature 463:311-317

Nei M, Niimura Y, Nozawa M (2008) The evolution of animal chemosensory receptor gene repertoires: roles of chance and necessity. Nat Rev Genet 9:951-963

Nelson G, Hoon MA, Chandrashekar J, Zhang Y, Ryba NJP, Zuker CS (2001) Mammalian sweet taste receptors. Cell 106:381-390

Nelson G, Chandrashekar J, Hoon MA, Feng L, Zhao G, Ryba NJP, Zuker CS (2002) An amino-acid taste receptor. Nature 416:199 202 
Posada D, Crandall KA (1998) MODELTEST: testing the model of DNA substitution. Bioinformatics 14:817-818

San Gabriel A, Uneyama H, Yoshie S, Torii K (2005) Cloning and characterization of a novel mGluR1 variant from vallate papillae that functions as a receptor for L-glutamate stimuli. Chem Senses $30: \mathrm{i} 25-\mathrm{i} 26$

Shi P, Zhang J (2006) Contrasting modes of evolution between vertebrate sweet/umami receptor genes and bitter receptor genes. Mol Biol Evol 23:292-300

Sonntag CF (1923) The comparative anatomy of the tongues of the Mammalia-VIII. Carnivora. Proc Zool Soc Lond 1923:129-153

Tamura K, Peterson D, Peterson N, Stecher G, Nei M, Kumar S (2011) MEGA5: Molecular Evolutionary Genetics Analysis using maximum likelihood, evolutionary distance, and maximum parsimony methods. Mol Biol Evol 28:2731-2739

Temussi PA (2009) Sweet, bitter and umami receptors: a complex relationship. Trends Biochem Sci 34:296-302
Yamaguchi S, Ninomiya K (2000) Umami and food palatability. J Nutr 130:921S-926S

Yarmolinsky DA, Zuker CS, Ryba NJP (2009) Common sense about taste: from mammals to insects. Cell 139:234-244

Yoshimura K, Shindoh J, Kobayashi K (2002) Scanning electron microscopy study of the tongue and lingual papillae of the California sea lion (Zalophus californianus californianus). Anat Rec 267:146-153

Zhao H, Yang J-R, Xu H, Zhang J (2010) Pseudogenization of the umami taste receptor gene Tas $1 r 1$ in the giant panda coincided with its dietary switch to bamboo. Mol Biol Evol 27:2669-2673

Zhao H, Xu D, Zhang S, Zhang J (2012) Genomic and genetic evidence for the loss of umami taste in bats. Genome Biol Evol 4:73-79

Zwickl DJ (2006) Genetic algorithm approaches for the phylogenetic analysis of large biological sequence datasets under the maximum likelihood criterion. $\mathrm{PhD}$ dissertation, University of Texas at Austin. http://repositories.lib.utexas.edu/handle/2152/2666. Accessed 21 June 2012 